\title{
Sequent Calculus and Equational Programming
}

\author{
(work in progress) \\ Nicolas Guenot and Daniel Gustafsson \\ IT University of Copenhagen \\ \{ngue, dagu\}@itu.dk
}

\begin{abstract}
Proof assistants and programming languages based on type theories usually come in two flavours: one is based on the standard natural deduction presentation of type theory and involves eliminators, while the other provides a syntax in equational style. We show here that the equational approach corresponds to the use of a focused presentation of a type theory expressed as a sequent calculus. A typed functional language is presented, based on a sequent calculus, that we relate to the syntax and internal language of Agda. In particular, we discuss the use of patterns and case splittings, as well as rules implementing inductive reasoning and dependent products and sums.
\end{abstract}

\section{Programming with Equations}

Functional programming has proved extremely useful in making the task of writing correct software more abstract and thus less tied to the specific, and complex, architecture of modern computers. This, is in a large part, due to its extensive use of types as an abstraction mechanism, specifying in a crisp way the intended behaviour of a program, but it also relies on its declarative style, as a mathematical approach to functions and data structures. However, the vast gain in expressivity obtained through the development of dependent types makes the programming task more challenging, as it amounts to the question of proving complex theorems - as illustrated by the double nature of proof assistants such as Coq [11] and Agda [18]. Keeping this task as simple as possible is then of the highest importance, and it requires the use of a clear declarative style.

There are two main avenues for specifying a language of proofs, or programs, that is abstract enough to support complex developments involving dependent types. The first approach, chosen by the Coq project, is to have a language of tactics that partially automate the construction of proofs - that is, to mechanically construct complex programs based on the composition of a few generic commands. While this takes the development task closer to the usual idea of proving a mathematical theorem, the second approach is to take the programming viewpoint: although Coq allows to directly write proof terms, this is better illustrated by Agda, where a syntax inspired by Haskell [1] provides a clear equational style.

Our goal here is to investigate the relations between the equational style of dependently-typed functional programming as found in Agda to the proof-theoretical description of intuitionistic logic given in the sequent calculus. In particular, we claim that a focused sequent calculus, akin to the LJF system of Liang and Miller [15], offers a logical foundation of choice for the development of a practical dependently-typed language. We intend to support this claim by showing how the equational syntax of Agda and the internal structure of its implementation correspond to a computational interpretation of such a calculus - for an extended for of intuitionistic logic including dependencies and (co)induction. As it turns out, the use of left rules rather than eliminations for positive connectives such as disjunction, in sequent calculus, yields a simpler syntax. In general, beyond the use of spines in applications, as in LJT [13] and quite common in the implementation of functional programming languages or proof

I. Cervesato and K. Chaudhuri (Eds.): Tenth International Workshop on Logical Frameworks and Meta-Languages: Theory and Practice EPTCS 185, 2015, pp. 102-109 doi 10.4204/EPTCS.185.7
This work is licensed under the Creative Commons Attribution License. 
assistants, the structure of the sequent calculus is much closer to the equational style of programming than natural deduction, the standard formalism in which type theory is usually expressed [16]. Using a focused system rather than a plain sequent calculus based on $\mathbf{L J}$ provides a stronger structure, and emphasizes the importance of polarities, already observed in type theory [2].

Beyond the definition of a logical foundation for a functional language in equational style, giving a proof-theoretical explanation for the way Agda is implemented requires to accomodate in the sequent calculus both dependent types and a notion of inductive definition. This is not an easy task, although there has been some work on dependent types in the sequent calculus [14] and there is a number of approaches to inductive definitions in proof theory, including focused systems [5]. For example, the system found in [14] is based on LJT but is limited to $\Pi$ and does not support $\Sigma$, while [12] has both, but requires an intricate mixture of natural deduction and sequent calculus to handle $\Sigma$. Induction is even more complex to handle, since there are several approaches, including definitions [19] or direct least and greatest fixpoints as found in $\mu$ MALL [5] and $\mu \mathbf{L J}$ [4]. From the viewpoint of proof-theory, the least fixpoint operator $\mu$ seems to be well-suited, as it embodies the essence of induction, while the greatest fixpoint $v$ allows to represent coinduction. However, these operators are not used the same way as inductive definitions found in Agda or other languages or proof assistants - they seem more primitive, but the encoding of usual constructs in terms of fixpoints is not obvious. Even more complicated is the question of using fixpoints in the presence of dependent types, and this has only been studied from the type-theoretic viewpoint in complex systems such as the Calculus of Inductive Constructions [10]. In the end, what we would like to obtain is a proof-theoretical understanding of the equational style of dependent and (co)inductive programming, related to the goals of the Epigram project. In particular, we consider that the sequent calculus, with its use of left rules, provides access to the "left" of equations in a sense similar to what is described in [17].

Here, we will describe the foundamental ideas for using a variant of $\mathbf{L J F}$ as the basis for the design of a dependently-typed programming language. We start in Section 2 by considering a propositional system and show how the shape of sequent calculus rules allows to type terms in equational style. This is made even more obvious by the use of pattern in the binding structure of the calculus. Then, in Section 3 we discuss the extension of this system to support dependent types and induction, problems related to patterns in this setting, as well as the question of which proof-theoretical approach to induction and coinduction is better suited for use in a such a language. Finally, we conclude by the review of some research problems opened by this investigation, and an evaluation of the possible practical applications to languages and proofs assistants.

\section{Focusing and Polarities in the Sequent Calculus}

We start our investigation with a propositional intuitionistic system presented as a focused sequent calculus. It is a variant of $\mathbf{L J F}$ [15] to which we assign a term language extending the $\bar{\lambda}$-calculus of Herbelin [13]. Unlike the calculus based on LJT, this system has positive disjunctions and conjunctions $\vee$ and $\times$, but it has no positive atoms. We use the following grammar of formulas:

$$
N, M::=a|\uparrow P| P \rightarrow N|N \wedge M \quad P, Q::=\downarrow N| P \vee Q \mid P \times Q
$$

where $\uparrow$ and $\downarrow$ are called polarity shifts and are meant to maintain an explicit distinction between the two categories of formulas, negatives and positives. This is not absolutely necessary, but it clarifies the definition of a focused system by linking the focus and blur rules to actual connectives. Note that this was also used in the presentation of a computational interpretation of the full $\mathbf{L J F}$ system [7]. 


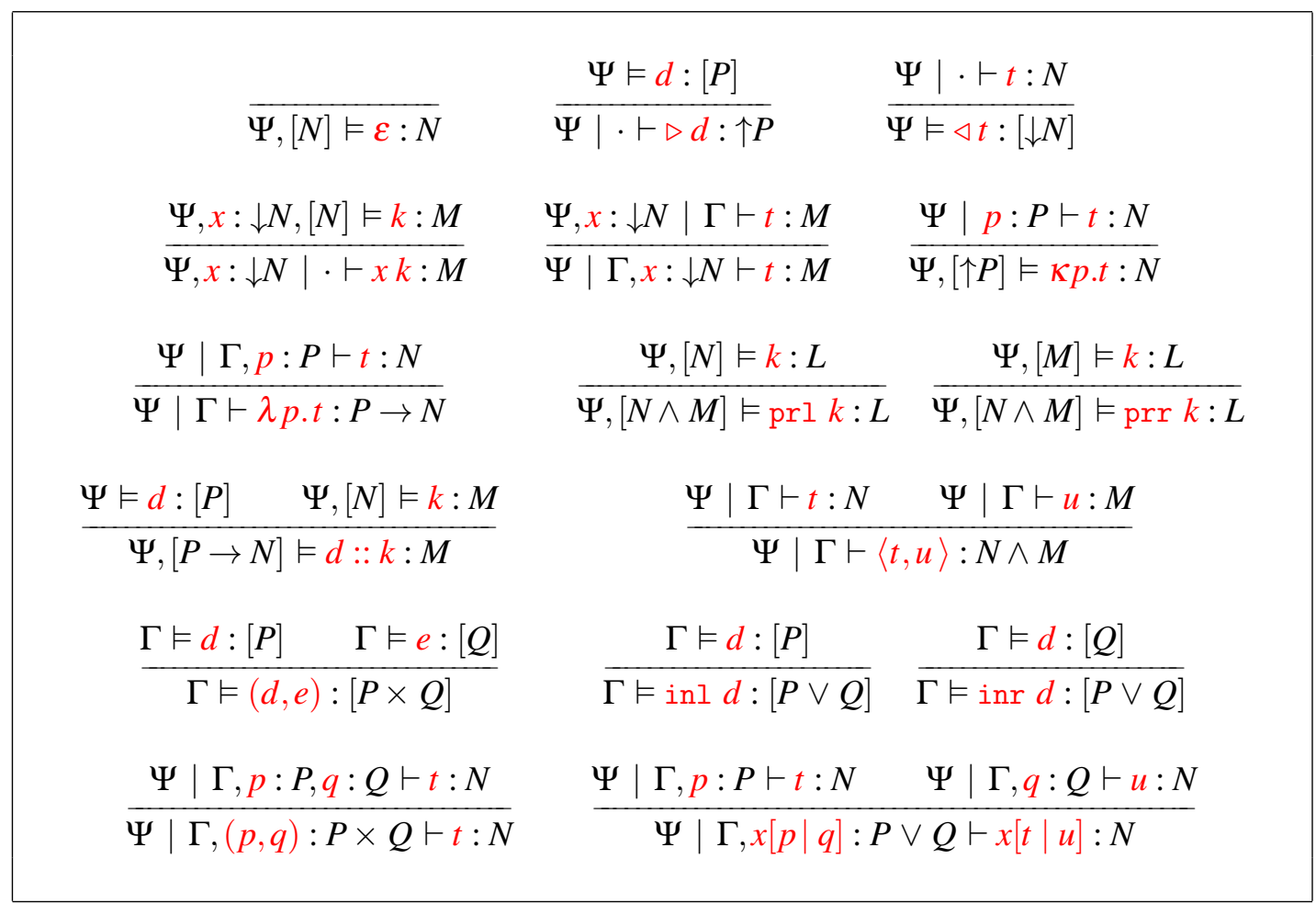

Figure 1: Typing rules for a pattern-based $\lambda$-calculus based on $\bar{\lambda}$

The rules we use in this system are shown in Figure 1, where the term assignment is indicated in red and several turnstiles are used to distinguish an inversion phase $\vdash$ from a focused phase $\vDash$. In this syntax, brackets are used to pinpoint the precise formula under focus. The extended $\lambda$-calculus we use to represent proofs is based on the following grammar:

$$
\begin{array}{rl|l|l|}
t, u & :=\triangleright d & \lambda p . t & x k|\langle t, u\rangle| x[t \mid u] \\
p, q::=x & (p, q) & x[p \mid q] \\
d, e::=\triangleleft t & (d, e) & \text { inl } d \mid \text { inr } d \\
k, m::=\varepsilon & t:: k & \operatorname{prl} k|\operatorname{prr} k| \kappa p . t
\end{array}
$$

where $t$ denotes a term, $p$ a binding pattern, $d$ a data structure and $k$ an application context. In terms of programming, terms are describing computation, mostly by means of functions, while data structures implement pairs and constructors. Note that computations can use case splittings $x[t \mid u]$ to choose between the subterms $t$ or $u$ depending on the contents of the data bound to $x$. The use of patterns rather than plain variables to annotate formulas in the context of typing judgement is taken from [8] and allows to express more directly the equational style found in Agda. For example, we could write:

$$
\begin{aligned}
& f:(\mathbb{N} \times \mathbb{N}) \uplus \mathbb{N} \rightarrow \mathbb{N} \\
& f(\operatorname{inl}(x, y))=x+y \\
& f(\operatorname{inr} z)=z
\end{aligned}
$$

to define a function $f$ that uses pattern-matching on its argument and computes the result based on the components of the data structure it received. Such a function can be written in our calculus as the following 
term: $\lambda w[(x, y) \mid z] \cdot w[$ add $((x \varepsilon)::(y \varepsilon):: \varepsilon) \mid z \varepsilon]$, where add is the name of the addition function. This makes the compilation of the code written above to the adequate representation in our calculus relatively easy, since different parts of a definition can be aggregated into a term with a pattern and a case splitting. This is very much related to the question of compiling pattern-matching into a specific splitting tree where case constructs are used [3].

The idea of the logical approach is that cut elimination in this system yields a reduction system implementing the dynamics of computation in the corresponding calculus. In such a focused calculus, a number of cut rules are needed to complete the proof of completeness of the cut-free fragment, but only two of them really need to be considered as rules - the other cuts can simply be stated as principles, and their reduction will correspond to a big step of computation. These two rules are:

$$
\frac{\Psi \vDash d:[P] \quad \Psi \mid \Gamma, p: P \vdash t: N}{\Psi \mid \Gamma \vdash p=d \text { in } t: N} \quad \frac{\Psi \mid \Gamma \vdash t: N \quad \Psi,[N] \vDash k: M}{\Psi \mid \Gamma \vdash t k: M}
$$

the first one being the binding of a data structure to a matching pattern, and the second a simple application of a term to a list of arguments. The latter is already part of the LJT system [13], but the former is specific to $\mathbf{L J F}$ in the sense that it appears only when formulas can be focused on the right of a sequent. The main reduction rule extracted from cut elimination is the $\bar{\lambda}$ variant of $\beta$-reduction:

$$
(\lambda p . t)(d:: k) \rightarrow(p=d \text { in } t) k
$$

but there are a number of other reduction rules generated by the use of other connectives than implication. In particular, conjunction yields a form of pairing where a term $\langle t, u\rangle$ has to be applied to a list prl $k$ to reduction to $t k$. The binding cut is simpler in a certain sense, since its reduction corresponds to a decomposition of the data structure $d$ according to the shape of the pattern $p$, and a simple substitution when $p$ is just a variable. Moreover, other cuts encountered during reduction usually amount to a form of substitution, except for the one, already present in LJT, that yields lists concatenation in the argument of an application.

Note that the $\triangleright d$ construct is present in the internal language of Agda, but the constructs $\triangleleft t$ and $\kappa p . t$ are not, although they can be obtained indirectly using a cut. While $\triangleleft t$ should simply be understood as a thunk, which is a term made into data, the list $\kappa$ p.t is slightly more complex. This construct, already present in [6], is more a context than a list in the sense that it stops the application of a term to $\kappa p . t$ and enforces the execution of $t$, where the original term applied is bound to $p$. This can be understood by considering the reduction extracted from cut elimination:

$$
(\triangleright d)(\kappa p . t) \rightarrow p=d \text { in } t
$$

Finally, note that we could have an explicit contraction rule in the system, that would appear in terms under the form of a pattern $p \Subset q$ indicating that $p$ and $q$ will be the patterns associated to two copies of the same assumption $P$. The associated typing rule is:

$$
\frac{\Psi \mid \Gamma, p: P, q: P \vdash t: N}{\Psi \mid \Gamma, p @ q: P \vdash t: N}
$$

and it is reminiscent of the pattern using the same syntax in Haskell - which is meant to exist in Agda as well, but this not yet implemented. However, in Haskell, this is restricted to the form $x @ p$ so that it can only serve to name an assumption before decomposing it, and we could allow for such a use by avoiding maximal inversion, which is not strictly necessary in a focused system [7]. This rule is not necessary for the completeness of the calculus, and there are other ways to obtain the same result. Of course, in a very similar way, the pattern _ can be associated to the weakening rule, also admissible. 


$$
\begin{aligned}
& \overline{\Psi,[N] \vDash \varepsilon: N} \quad \frac{\Psi \vDash d:[P]}{\Psi \mid \cdot \vdash \triangleright d: \uparrow P} \quad \frac{\Psi \mid \cdot \vdash t: N}{\Psi \vDash \triangleleft t:[\downarrow N]} \quad \frac{\Psi,[N] \vDash k: L}{\Psi,[N \wedge M] \vDash \operatorname{prl} k: L} \\
& \frac{\Psi, x: \downarrow N,[N] \vDash k: M}{\Psi, x: \downarrow N \mid \cdot \vdash x k: M} \quad \frac{\Psi, x: \downarrow N \mid \Gamma \vdash t: M}{\Psi \mid \Gamma, x: \downarrow N \vdash t: M} \quad \frac{\Psi \mid x: P \vdash t: N}{\Psi,[\uparrow P] \vDash \kappa x . t: N} \quad \frac{\Psi,[M] \vDash k: L}{\Psi,[N \wedge M] \vDash \operatorname{prr} k: L} \\
& \frac{\Psi \mid \Gamma, x: P \vdash t: N}{\Psi \mid \Gamma \vdash \lambda x . t: \Pi(x: P) . N} \frac{\Psi \vDash d:[P] \quad \Psi,[N\{d / x\}] \vDash k: M}{\Psi,[\Pi(x: P) \cdot N] \vDash d:: k: M} \frac{\Psi|\Gamma \vdash t: N \quad \Psi| \Gamma \vdash u: M}{\Psi \mid \Gamma \vdash\langle t, u\rangle: N \wedge M} \\
& \frac{\Psi \mid \Gamma, y: P, z: Q \vdash t: N\{(y, z) / x\}}{\Psi \mid \Gamma, x: \Sigma(y: P) \cdot Q \vdash y, z=x \operatorname{in} t: N} \quad \frac{\Gamma \vDash d:[P]}{\Gamma \vDash(d, e):[\Sigma(x: P) \cdot Q]} \quad \frac{\Gamma \vDash d:[P]}{\Gamma \vDash \operatorname{inl} d:[P \vee Q]} \\
& \frac{\Psi|\Gamma, y: P \vdash t: N\{\operatorname{inl} y / x\} \quad \Psi| \Gamma, z: Q \vdash u: N\{\operatorname{inr} z / x\}}{\Psi \mid \Gamma, x: P \vee Q \vdash x[y . t \mid z . u]: N} \quad \frac{\Gamma \vDash d:[Q]}{\Gamma \vDash \operatorname{inr} d:[P \vee Q]} \\
& \frac{\Psi \vDash d:[A] \quad \Psi \mid \Gamma, x: A, \Delta \vdash t: B}{\Psi \mid \Gamma, \Delta\{d / x\} \vdash x=d \text { in } t: B\{d / x\}} \quad \frac{\Psi \mid \Gamma \vdash t: A \quad \Psi,[A] \vDash k: B}{\Psi \mid \Gamma \vdash t k: B}
\end{aligned}
$$

Figure 2: Typing rules for a dependent $\lambda$-calculus based on $\bar{\lambda}$

\section{Adding Dependent Types and Induction}

We continue our investigation by adapting our variant of LJF to dependent types, but this unveils some issues that we will now discuss. On problem we immediately encounter is the adaptation of the pattern machinery to the dependent setting, mostly due to the substitutions involved in the types, where patterns should have appeared. For the dependent implication $\Pi(x: P) \cdot N$, using a pattern $p$ rather than a binding variable $x$ yields the question of substituting a data structure $d$ for $p$ : this becomes a much more complicated operation than the traditional substitution. Moreover, keeping the patterns and variables synchronised between their use in terms and in types is a challenging task, that would probably require heavy syntactic mechanisms. For this reason, the system shown above in Figure 2 has no patterns, but rather falls back to the traditional style of typing using only variables to label assumptions. The language used in this variant can still be related to the equational approach to functional programming, but the translation between equations and terms is more involved.

The generalisation of the implication into the dependent product $\Pi(x: P) . N$ is a straightforward operation, and the rules we use are essentially the ones found in [14] - except that it involves a data structure, corresponding to a focus on the right-hand side of a sequent. Now, the case of $\Sigma$ is more complicated, as it is a priori unclear whether it should be obtained as a generalisation of the negative conjunction $\wedge$ or of the positive product $\times$ and both solutions might even be possible. But a generalisation of the negative disjunction seems to be problematic, when it comes to the specification of the second left rule, typing the prroperation. Indeed, when focusing on $\Sigma(x: N) . M$ we would need to plug a term of type $N$ for $x$ in $M$, but this would require to maintain some "natural deduction version" of the term currently 
being transformed, and to plug at adequate locations some translation between natural deduction style and our sequent calculus syntax - as done in [12]. This is quite unsatisfactory and will not help us build a proper understanding of dependent types in a pure sequent calculus setting. The solution we adopt here is to obtain $\Sigma(x: P) \cdot Q$ as a generalisation of the positive product $\times$ and simply update the corresponding rules as shown in Figure 2. The left rule is simple to define in this case, because the decomposition of the $\Sigma$ in the context preserves the binding of $y$ in the type $Q$.

There is a particularly interesting benefit to the use of the sequent calculus to handle splitting as done in the left $\Sigma$ rule. Consider the elimination rule in natural deduction:

$$
\vee \boldsymbol{e} \frac{\Gamma, x: A \vee B \vdash C: \text { type } \quad \Gamma \vdash t: A \vee B \quad \Gamma, y: A \vdash u: C\{\operatorname{inl} y / x\} \quad \Gamma, z: B \vdash v: C\{\operatorname{inr} z / x\}}{\Gamma \vdash \operatorname{match}[x . C](t ; y . u ; z . v): C\{t / x\}}
$$

and observe that it is necessary to be explicit about the return type, since obtaining $C$ from $C\{t / x\}$ is a complicated process, that reverses a substitution. This makes the term syntax heavy, while the problem is avoided in the sequent calculus, where no substitution is needed in the conclusion. Note that in Coq, the natural deduction style is used for the proof language, but tactics are written in a style that is much closer to the sequent calculus - as this is the framework of choice for proof search - so that tactics have to perform some kind of translation between the two formalisms.

At the level of dependent types, there is a number of tricks used in the Agda implementation that diverge from the proof-theoretical viewpoint. For example, substitutions in types are treated in a complex way and may be grouped together. Although some of the design choices can be justified by a similarity to the focused sequent calculus, there is probably a number of implementation techniques that have no proof-theoretical foundation. Moreover, we have chosen here a particularly precise framework where formulas are explicitly polarised, but in practice types in a programming language should not always require these annotations: the question of the presence of specific terms corresponding to shifts is therefore not obvious, as it depends if some interesting programming constructs require their presence or their absence. One can observe, for example, that in the system proposed here, dependencies are subject to the presence of delays, because of the contraction present in the left focus rule and of the treatment of names in the $\kappa x$.t operation.

The problem of generalising the equational style of programming associated to the focused sequent calculus at the propositional level to the level of dependent types is parametrised by a choice: using patterns seems to require a complex tracking mechanism, but provides a relatively direct logical representation of equations, while using simple variables leads to a translation overhead. Notice however that one could think of an implementation based on variables in which equations are easily obtained, since the language would already be expressed in the style of the sequent calculus - this is the approach suggested by Epigram, where equations are meant to clarify the meaning of programs but are not their internal representation. But we now turn to the most challenging task of our whole enterprise: the accomodation of induction in the framework of a focused sequent calculus in a form that can be exploited to design declarative programs.

Induction can be expressed in Agda in a concise manner and enjoys the benefits of the equational presentation. Consider for example the following inductive scheme for natural numbers:

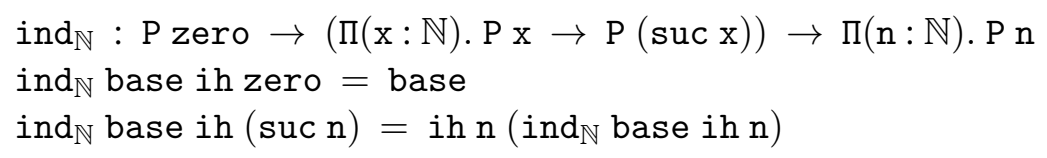

where the code essentially relies on the matching of a natural number, that can be either zero or the successor of another number. It is not obvious to see through this program and select a particular approach 
to induction that would be a good candidate for a proof-theoretical description. The natural candidate for a representation of induction in the sequent calculus would be the $\mu$ operator as studied in [4] in the setting of intuitionistic logic. The unfocused rules for this operator would be, from a purely logical viewpoint:

$$
\frac{\Gamma \vdash B\{\mu a . B / a\}}{\Gamma \vdash \mu a . B} \quad \frac{B\{C / a\} \vdash C}{\Gamma, \mu a \cdot B \vdash C}
$$

but the presence of fixpoints has consequences for cut elimination, as it prevents some cuts to be reduced. The usual technique applied to avoid this problem is to build the cut rule into the left rule for $\mu$ and to consider the result as cut free. This way, all the cuts that cannot be reduced further are explicitly attached to the blocking rule instance. However, the use of these rules in terms of computation is not obvious to specify, in part because of the complexity of the associated cut reduction, that involves the creation of several other cuts and appeals to the functoriality of the body $B$ of any fixpoint $\mu a . B$ - ensured by a positivity condition. In addition, these rules seem to interact poorly with dependent types, as dealing with fixpoints will require a complex handling of terms appearing inside types. It is unclear as of now if fixpoints as expressed by $\mu$ - and $v$ in the case of induction - can fit our scheme of explaining the implementation of a language such as Agda, but at the same time there is no obvious proof-theoretical approach that accounts in a straightforward way for the pervasive nature of inductive definitions in the internal language of Agda, where they are handled by expansion of names with the body of the definition.

\section{Conclusion and Future Work}

As we have seen here, the $\bar{\lambda}$-calculus proposed by Herbelin as an interpretation of the LJT focused sequent calculus can be extended beyond its original scope to include positive connectives, leading to a full-fledged intuitionistic system where we can focus on the right-hand side of sequents to decompose positives. The language we obtain is well-suited to represent programs written in the kind of equational style found in Haskell or Agda, the relation to equations can be made even tighter by using patterns as labels for assumptions in the type system. The opens up the possibility to select focused sequent calculus as a logical framework of choice for the implementation of such languages - as evidenced by the current state of the implementation of Agda, containing many elements that can be explained as sequent calculus constructs. The benefit could not only be a simplication of such an implication, but possibly an improvement in terms of efficiency if advanced techniques from proof theory are transferred and made practical. Moreover, one of the strength of the logical approach is that generalisations and extensions of all kinds are usually made simpler by the strong principles at work: any kind of progress made on the side of proof theory could translate into more expressive languages using the clear equational style of Haskell and Agda — that could be modalities, linearity or many other elements studied in the field of computational logic.

The generalisation of this idea to handle dependent types has already been partially investigated, but some question are left unresolved as to the specific rules used in such a system, and the possibility of making the system more equational by exploiting patterns. But the most difficult task at hand is the explanation of the various treatments of induction available in language and proofs assistants in terms of the sequent calculus. As observed previously [2], the notion of polarity seems to be important in the understanding of this question, but unfortunately the proper polarised handling of fixpoints in proof theory has yet to be found - a number of choices are left open when it comes to the definition of a focused system using fixpoints [5]. Note that our enterprise also yields the question of the treatment of the identity type in proof theory, as it makes dependent pattern matching admit the axiom $\boldsymbol{K}$. This axiom is undesirable 
in homotopy type theory, and thus the restriction of dependent pattern matching has been studied [9]. But this was achieved by restricting unification in the splitting rules, and as Agda has no explicit calculus for splitting, this was somewhat hidden. The framework we want to develop provides a calculus and could thus help making this restriction simpler.

Acknowledgements. This work was funded by the grant number 10-092309 from the Danish Council for Strategic Research to the Demtech project.

\section{References}

[1] Haskell, an advanced purely-functional programming language: http://www.haskell.org.

[2] Andreas Abel, Brigitte Pientka, David Thibodeau \& Anton Setzer (2013): Copatterns: programming infinite structures by observations. In: POPL'13, pp. 27-38, doi $10.1145 / 2429069.2429075$.

[3] Lennart Augustsson (1985): Compiling Pattern Matching. In: FPCA'85, pp. 368-381, doi:10.1007/3-540-15975-4_48.

[4] David Baelde (2008): A linear approach to the proof-theory of least and greatest fixed points. Ph.D. thesis, Ecole Polytechnique.

[5] David Baelde (2012): Least and Greatest Fixed Points in Linear Logic. ACM Transactions on Computational Logic 13(1), p. 2, doi $10.1145 / 2071368.2071370$.

[6] Henk Barendregt \& Silvia Ghilezan (2000): Lambda-terms for natural deduction, sequent calculus and cut elimination. Journal of Functional Programming 10(1), pp. 121-134.

[7] Taus Brock-Nannestad, Nicolas Guenot \& Daniel Gustfasson (2015): Computation in Focused Intuitionistic Logic. In: PPDP'15, pp. 43-54, doi:10.1145/2790449.2790528

[8] Serenella Cerrito \& Delia Kesner (1999): Pattern Matching as Cut Elimination. In: LICS'99, pp. 98-108, doi $10.1109 /$ LICS.1999.782596.

[9] Jesper Cockx, Dominique Devriese \& Frank Piessens (2014): Pattern Matching Without K. In: ICFP'14, pp. 257-268, doi:10.1145/2628136.2628139.

[10] Thierry Coquand \& Christine Paulin (1988): Inductively defined types. In: Conference on Computer Logic, LNCS 417, pp. 50-66, doi $10.1007 / 3-540-52335-9 \_47$.

[11] Gilles Dowek, Amy Felty, Gérard Huet, Hugo Herbelin, Chet Murthy, Catherine Parent, Christine Paulin-Mohring \& Benjamin Werner (1993): The Coq proof assistant user's guide. Technical Report, INRIA.

[12] Roy Dyckhoff \& Luís Pinto (1998): Sequent Calculi for the Normal Terms of the $\lambda \Pi$ - and $\lambda \Pi \Sigma$-Calculi. Electronic Notes in Theoretical Computer Science 17, pp. 1-14, doi:10.1016/S1571-0661(05)01182-5.

[13] Hugo Herbelin (1994): A $\lambda$-Calculus Structure Isomorphic to Gentzen-Style Sequent Calculus Structure. In L. Pacholski \& J. Tiuryn, editors: CSL'94, LNCS 933, pp. 61-75, doi 10.1007/BFb0022247

[14] Stéphane Lengrand, Roy Dyckhoff \& James McKinna (2011): A Focused Sequent Calculus Framework for Proof Search in Pure Type Systems. Logical Methods in Computer Science 7(1), doi:10.2168/LMCS-7(1:6)2011.

[15] Chuck Liang \& Dale Miller (2009): Focusing and Polarization in Linear, Intuitionistic, and Classical Logics. Theoretical Computer Science 410(46), pp. 4747-4768, doi 10.1016/j.tcs.2009.07.041.

[16] Per Martin-Löf (1984): Intuitionistic Type Theory. Studies in Proof Theory, Bibliopolis.

[17] Conor McBride \& James McKinna (2004): The view from the left. Journal of Functional Programming 14(1), pp. 69-111, doi: $10.1017 /$ S0956796803004829

[18] Ulf Norell (2007): Towards a practical programming language based on dependent type theory. Ph.D. thesis, Chalmers University of Technology.

[19] Peter Schroeder-Heister (1993): Rules of Definitional Reflection. In M. Vardi, editor: LICS'93, pp. 222-232, doi $10.1109 /$ LICS.1993.287585. 